Original Article

\title{
A study of functional outcome of laminectomy and discectomy in lumbar intervertebral disc prolapse (a comparison of retrospective and prospective analysis)
}

\section{Sanath Kumar Shetty ${ }^{1}$, Arjun Ballal ${ }^{2}$, Lawrence John Mathias ${ }^{3} \&$ H. Ravindranath Rai ${ }^{4}$}

${ }^{1}$ Assistant Professor, ${ }^{3}$ Professor, ${ }^{4}$ Professor \& HOD, Department of Orthopaedic Surgery,

K. S. Hegde Medical Academy, M angalore, ${ }^{2}$ Registrar, Department of Orthopaedic surgery, ARS Hospital, Tirupur.

\section{Correspondence}

Sanath Kumar Shetty

Assistant Professor, Department of Orthopaedic Surgery, K.S. Hegde M edical Academy, M angalore - 575 018, Karnataka, India. Mobile : +91 9845069383 E-mail : sanathkumarshetty@hotmail.com

\section{Abstract}

Background: Back pain is considered as one of the most unrewarding problems in clinical medicine. There have existed several etiologies for the same and most of them do not have an ideal clinical presentation. Only those syndromes associated with neurologic compression of the cauda equina or nerve roots, have reasonably well understood clinical presentation.

Aims: The aim of the study was to analyse and compare the functional outcome of laminectomy and discectomy in lumbar intervertebral disc prolapse in short and long term follow up.

Materials and methods: Our study included a total of 50 diagnosed cases of lumbar intervertebral disc prolapse between age group of 40-70 years. The patients were subdivided into two groups. The first group was a retrospective group and included patients who had undergone laminectomy and discectomy between January 1993 and December 2003 with an average follow up of 3.68 years. Group two (prospective group) consisted of patients between January 2004 and December 2004 who underwent laminectomy and discectomy, with a follow up of 6 months.

The subjective assessment was done using the Oswestry disability index (O.D.I). the results obtained with assessment of group I and group II were tabulated compared. Statistical analysis was done using the chi square test.

Results: The results were noted to be $76 \%$ excellent, $8 \%$ good and $16 \%$ with poor scores in group I. $96 \%$ with excellent, no patients with good scores and $4 \%$ with poor scores in group II as per the O.D.I.

Conclusion: We conclude saying that laminectomy and discectomy had excellent outcome in terms of pain relief in terms of long term and short term follow up.

Keywords: Laminectomy, lumbar disc prolapse, Oswestry disability index, radiculopathy, cauda equina

\section{Introduction}

Lumbar intervertebral disc prolapse requiring medical attention in on the rise day by day ${ }^{1}$. It is said to have an incidence in almost $80 \%$ of the adults in some point of their life. Among chronic conditions, back problems are most frequently the cause for limitation of activity².

\begin{tabular}{|c|}
\hline Access this article online \\
\hline Quick Response Code \\
\hline
\end{tabular}

Medical or surgical management, the major objective is improvement in the functional outcome of the patient ${ }^{3}$.

Laminectomy and discectomy is a common procedure performed for the management of intervertebral disc prolapse. In most of the reports the functional outcome and the neurological recovery do show striking variations. This can be due to several factors like patient selection, surgical technique etc ${ }^{2}$.

Measurement of spine function can be accomplished via many standardises techniques such as Oswestry Disability Index (ODI), Visual Analog Scales (VASB and VASL), RolandM orris Disability Questionnaire etc ${ }^{4,5}$.

Aims: To assess and compare the functional outcome after laminectomy and discectomy of lumbar intervertebral disc prolapse in short term and long term follow ups. 


\section{Materials and methods.}

A total of 50 diagnosed cases of lumbar intervertebral disc prolapse were enrolled in the study. The patients were divided into two groups. The first group or the retrospective group included 25 patients who had undergone laminectomy and discectomy in the time range extending from January 1993 and December 2003. The follow up period was a mean of 3.63 years. The second group or the prospective group included a total of 25 diagnosed cases of lumbar intervertebral disc prolapse who underwent laminectomy and discectomy. The inclusion criteria for selection of cases included, patients with degenerative lumbar intervertebral disc prolapse (IVDP) between the age of 40 to 70 years. Traumatic and other causes of IVDP were excluded from the study. Complete neurological and radiological examination was done in the second group.

The surgical procedure $^{6}$ included a midline posterior approach to the spine (Figure 1). Once the lamina was exposed laminectomy was performed. The level of the

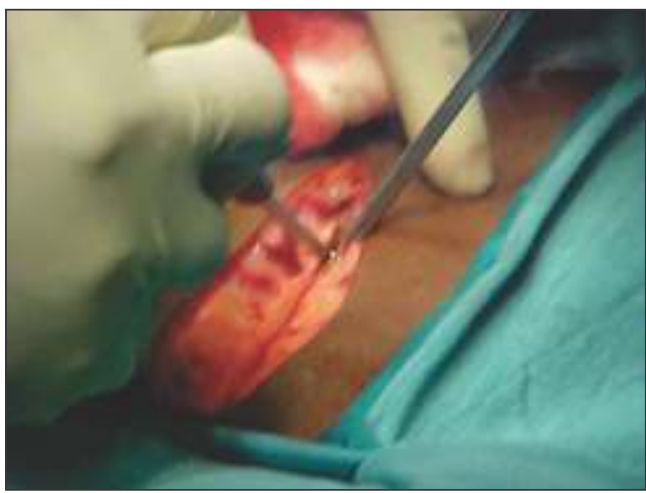

Figure I: Showing the midline posterior exposure to the lumbar spine.

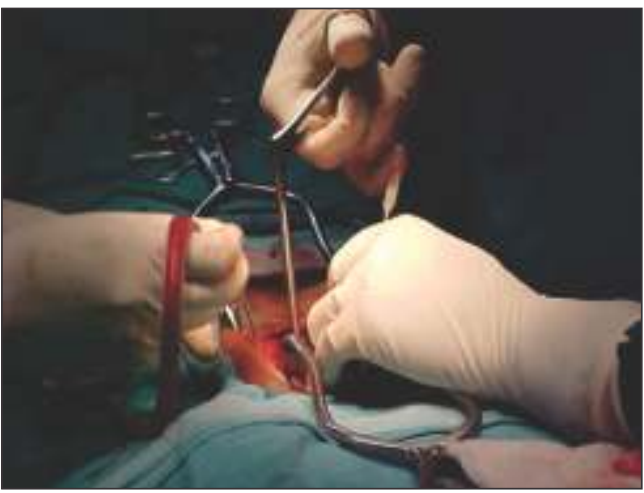

Figure 2 : Laminectomy being performed.

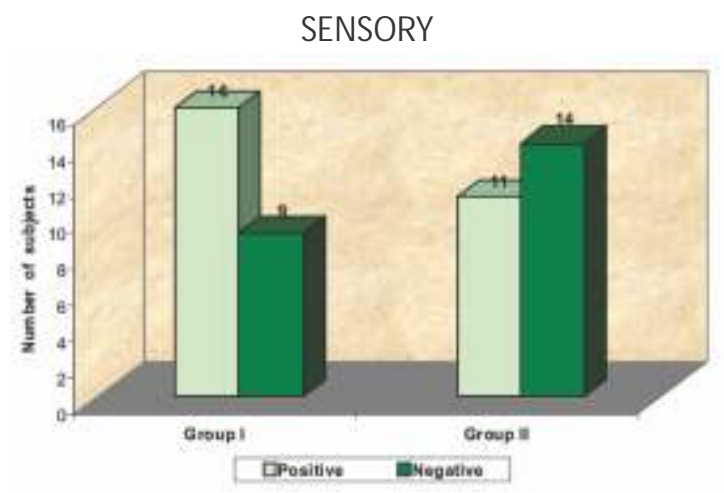

Graph I : Bar graph indicating the distribution of patients as per the presence or absence of sensory deficits. 16 patients group I had sensory deficits rest did not have sensory deficits. No motor deficits were noted in our patients. 11 patients (44\%) had sensory deficits and 14 (56\%) did not have sensory deficits in group II (Graph I).

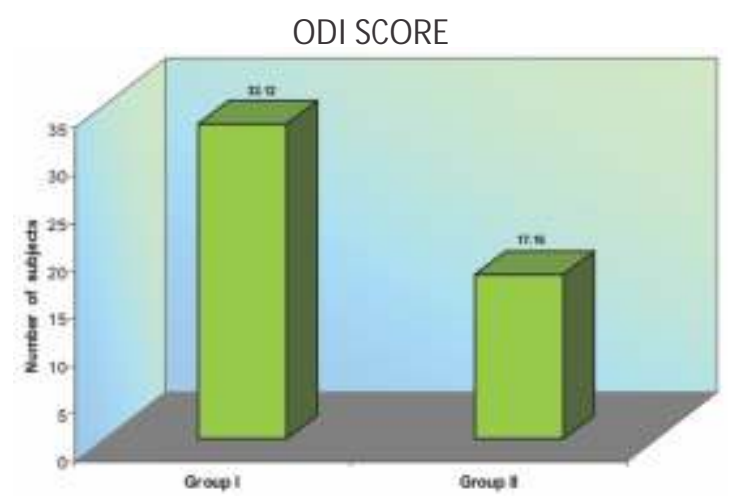

Graph II : Bar graph indicating the mean calculated in both the groupsas per the ODI scores.

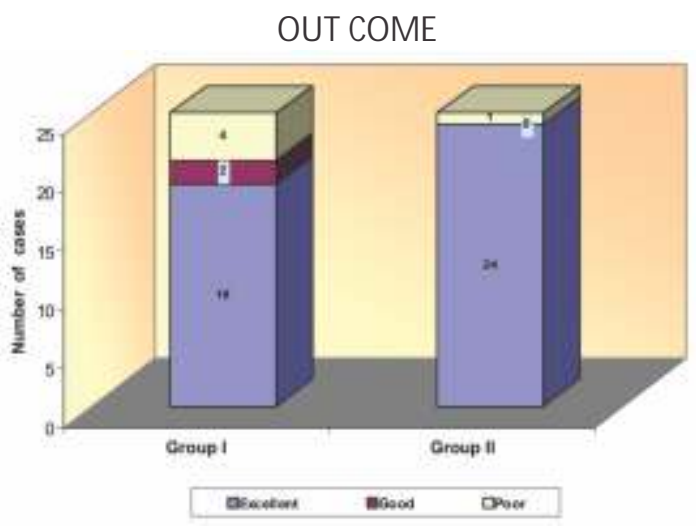

Graph III : Bar graph indicating the distribution of patients as per the inference of the ODI scores. 19 patients (76\%) had excellent outcome following surgery, $2(8 \%)$ had good outcome and 4 (16\%) had poor outcome in group I. 24 patients (96\%) had excellent outcome following surgery and $1(4 \%)$ had poor outcome, none of them had good outcome (Graph III). 
intervertebral disc was confirmed under fluroscopic guidance and retrieval of disc through disc forceps was done (Figure 2). Complete closure of the surgical site was done and patient was advised complete bed rest for 2 days and was advised mobilization with a brace after two days.

The patients were discharged on day ten after surgery after suture removal. They were reviewed at three, six, twelve weeks and then at six months. At six months the functional outcome was assessed as per the Oswestry disability index (O.D.I) $)^{7,8}$.

In the retrospective group the ODI scoring was done during the review. The results were tabulated and compared.

Statistical analysis was done using 'chi-square test'.

\section{Results}

19 of the 25 patients in group I were males and rest were females. In group II, 17 were males and rest were females. 16 patients group I had sensory deficits rest did not have sensory deficits. No motor deficits were noted in our patients. 11 patients (44\%) had sensory deficits and 14 (56\%) did not have sensory deficits in group II (Graph I). The mean ODI scores was noted to be 33.12 in group I and 17.16 in group II (Graph II). 19 patients (76\%) had excellent outcome following surgery, 2(8\%) had good outcome and 4 (16\%) had poor outcome in group I. 24 patients (96\%) had excellent outcome following surgery and 1 (4\%) had poor outcome, none of them had good outcome (Graph III). No complications were noted in any of the cases in either of the groups.

\section{Discussion}

Weber in 1983 expressed that, disc herniation is a collective term, to describe a process with rupture of annulus fibrosus and subsequent displacement of the central mass of the disc into the intervertebral space, common to the dorsal or laterodorsal aspect of the disc ${ }^{9}$.

Magnetic resonance imaging offers increased soft tissue resolution and allow for evaluation of lateral recess pathology, in addition to visualizing the thoraco-lumbar region for possible spinal tumors. Modic M.T and coworkers in 1986 investigated the accuracy of MRI, metrizamide myelography (MM), and $\mathrm{CT}$ in lumbar disc disease. Their studies showed that M RI was more accurate than M M $(82.3 \%$ versus $71.4 \%)$ and was equal to CT ( $82.3 \%$ vs. $83 \%$ ) in diagnosis of disc herniations. They concluded that the combination of $\mathrm{MRI}$ and $\mathrm{CT}$ was equal in diagnostic accuracy to the combination of CT and MM $(92.5 \%$ vs. $89.4 \%)^{10}$.

In our study we used the ODI questionnaire for evaluation of functional outcome in our patients.

Loupasis G.A. in 1999 conducted a retrospective study evaluating seven to twenty year outcome of lumbar discectomy by a mailed self report questionnaire. Subjective disability was assessed by the Oswestry questionnaire. They found that the long term results of standard lumbar discectomy were not very satisfying ${ }^{11}$. However, in our study we noted good to excellent results in most of our cases in either of the groups for laminectomy and discectomy.

Herron L, Turner J in 1996 performed a prospective study regarding patient selection for lumbar discectomy with a revised objective rating system based on the severity of findings in each of 4 categories (neurologic signs, root tension signs, imaging findings, psychosocial environment).They found that the objective rating score was highly predictive of patient outcome at follow up with more than $80 \%$ of the patients having good results ${ }^{12}$.

Padua R et al; reported good results for 10-15 year follow ups of laminectomy and discectomy done for 150 cases $^{13}$.

\section{Conclusion}

Laminectomy and discectomy is an excellent procedure for management of degenerative intervertebral disc prolapse. 


\section{References}

1. Khoo LT, Fessler RG (2002) Microendoscopic decompressive laminotomy for the treatment of lumbar stenosis. Neurosurgery 51 : S146-154.

2. Mittal A, A. Chandrasekhar, Ram M ohan R, Ramprasad Rallapalli R, Y. Siva Prasad Y. Analysis of the Functional Outcome of Discectomy in Lumbar Disc Prolapse. IOSR Journal of Dental and M edical Sciences. Volume 14, Issue 5 Ver. VI (M ay. 2015), PP 73-80.

3. Knight Reginald Q, Scribani M , Krupa N, Grainger S, Goldberg C, Spivak Cand Jenkins P. Lumbar Decompressive Laminectomy or Laminotomy for Degenerative Conditions: Outcome Comparison of Traditional Open versus Less Invasive Techniques. Spine 2013, PP 1-10.

4. Parker SL, Adogwa O, Davis BJ, Fulchiero E, Aaronson O, et al. (2011) Cost- utility Analysis of Minimally Invasive Versus Open Multilevel Hemilaminectomy for Lumbar Stenosis. J Spinal Disord Tech 26: 42-47.

5. Parker SL, Fulchiero EC, Davis BJ, Adogwa O, Aaronson OS, et al. (2011) Cost- effectiveness of multilevel hemilaminectomy for lumbar stenosis-associated radiculopathy. Spine J 11: 705-711.

6. Yorimitsu E, Chiba K, Toyama Y, Hirabayashi K. Long term outcomes of standard discectomy for lumbar disc herniation: A follow up study of more than 10 years. Spine (Phila Pa 1976) 2001; 26(6): 652-657.

7. Fairbank JC, Pynsent PB. The Oswestry Disability Index. Spine. 2000; 25(22):2490-2452.

8. Fairbank JC, Couper J, Davies JB. The Oswestry low back pain questionnaire. Physiotherapy 1980;66:271-271.

9. Weber H. Lumbar disk herniation - A controlled prospective study with 10 years of observation. Spine, 1983; $8: 131$ - 140.

10. Modic MT, Masaryk T, Boumphrey F, et al.: Lumbar herniated disc disease and canal stenosis: Prospective evaluation by surface coil M R CT and M yelography. AJNR 7 : 709 - 717, 1986.

11. Loupasis, George A et al : Seven to twenty year outcome of lumbar discectomy: Spine. November 15, 1999; 24 (22) : 2313

12. Herron, Larry $D$; Turner, Judith A et al : Patient selection for lumbar discectomy with revised objective rating system : clinical orthopaedics and related research, April 1996; (325) : 148 - 155.

13. Padua R, Padua S, Romanini E, Padua L, de Santis E. Ten- to 15-year outcome of surgery for lumbar disc herniation: radiographic instability and clinical findings. Eur Spine J. 1999;8(1):70-4. 